\title{
Utilización de instrumentos de concha durante el Mesolítico y Neolítico inicial en contextos litorales de la región cantábrica: programa experimental para el análisis de huellas de uso en materiales malacológicos
}

\author{
Using shell tools in Mesolithic and early Neolithic coastal sites from Northern Spain: \\ experimental program for use wear analysis in malacological materials
}

\author{
David Cuenca Solana (*) \\ Ignacio Clemente Conte $(* *)$ \\ Igor Gutiérrez Zugasti (*)
}

\section{RESUMEN}

Uno de los debates más extendidos en la historiografía sobre el Mesolítico y el Neolítico inicial en la región cantábrica es el de la escasez de tecnologías "tradicionales" en la mayor parte de los contextos existentes, especialmente en aquellos con grandes acumulaciones de conchas. Actualmente, varias de las hipótesis propuestas atribuyen este fenómeno a diferencias en la organización espacial de los asentamientos, al aumento en la utilización de materiales perecederos o a cambios en las estrategias de subsistencia. A partir del hallazgo de siete instrumentos de concha en el yacimiento de Santimamiñe (Kortezubi, Bizkaia), que a su vez constituyen la primera evidencia de su categoría en la región cantábrica, se propone como hipótesis el empleo de tecnologías de concha en algunas de las actividades productivas desarrolladas por los grupos de cazadores recolectores de los períodos indicados. Con el objetivo de confirmar/refutar los resultados obtenidos mediante el análisis funcional de estos instrumentos se ha llevado a cabo un programa experimental con diferentes especies de moluscos para procesar madera, piel fresca/seca y planta no leñosa. Los resultados del programa experimental confirman la utilización de estos instrumentos en diversas actividades productivas orientadas al procesado de algunas de estas materias.

(*) Instituto Internacional de Investigaciones Prehistóricas de Cantabria (IIIPC). Universidad de Cantabria. Edif. Interfacultativo, Avda. Los Castros s/n. 39005 Santander, Cantabria. Correos electrónicos: cuencad@unican.es, igorgutierrez.zug@gmail.com

(**) Departamento de Arqueología y Antropología. IMFCSIC. C/Egipcíaques 15. E-08001 Barcelona. Correo electrónico: ignacio@imf.csic.es

Recibido: 2-XII-2009; aceptado: 20-I-2010.

\begin{abstract}
One of the most common debates surrounding the Mesolithic and early Neolithic periods in northern Spain focuses on the scarcity of lithic and osseous technologies identified in large shell midden contexts. Currently, several hypotheses have been proposed that attribute this phenomenon to differences in site spatial organization, increases in perishable material use, or changes in subsistence strategies. However, recently shell tools have been identified in the early Neolithic levels at Santimamine cave located in the Basque Country of northern Spain. These artifacts are the first evidence of shell tools to be identified in Northern Spain in an early Neolithic shell midden context. This paper proposes the hypothesis that shell tools were being used in subsistence activities. To test this hypothesis, the authors developed an experimental programme using different types of mollusc shells to examine evidence of functional use on wood, dry/fresh animal skin and non-woody plants. The experimental results were then used to examine the patterns of use on the seven shell tools from Santimamiñe. The results of the comparisons indicate that the seven shell tools have similar use patterns as the experimental shells. This evidence supports the proposed hypothesis that shell tools may have been used frequently in shell midden contexts during the Mesolithic and early Neolithic for the working of wood, plants or animal skin.
\end{abstract}

Palabras clave: Análisis funcional; Instrumentos de concha; Arqueología experimental; Arqueomalacología; Cazadores-recolectores; Región cantábrica.

Key words: Functional analysis; Shell tools; Archaeomalacology; Hunter-gatherers; Cantabrian region. 


\section{INTRODUCCIÓN}

Uno de los debates historiográficos sobre el período Mesolítico en la región cantábrica se ha centrado en la escasez de tecnologías recuperadas en algunos de los contextos arqueológicos, especialmente de la zona occidental (Arias 1992a; Clark 1976; González Morales 1982; González Morales et al. 2004). Esta escasez de "utillaje tradicional", es decir lítico y óseo, ha fomentado continuos debates acerca de la funcionalidad y uso que dieron los grupos mesolíticos a estos espacios de acumulación de restos malacológicos. A su vez, allí raramente se documentan indicios claros que permitan definirlos como lugares de hábitat. Suscitan de esta forma un doble debate: cuál fue la tecnología empleada por estos cazadores recolectores para desarrollar sus actividades productivas, y dónde las llevaron a cabo. Como respuesta a la baja presencia cuantitativa de utillaje lítico y óseo en algunos de estos contextos, se ha apuntado la posible incidencia de tecnologías confeccionadas con materiales perecederos, como la madera, en la realización de las actividades productivas (Arias 1992b; Clark 1976; González Morales 1995). Desgraciadamente, este tipo de materiales son difíciles de documentar de manera directa en los registros cantábricos. La única posibilidad de rastreo se basa en las evidencias indirectas a partir de indicadores como el análisis de las huellas de uso sobre el utillaje conservado. Respecto a estos contextos asturienses de la zona occidental, en el Mesolítico de la zona oriental tecnología lítica y ósea están mejor documentadas. Esta divergencia paralelamente se ve acompañada por una menor acumulación de recursos malacológicos en los contextos litorales orientales, y una representación de actividades productivas que parece mostrar una ocupación más estable de estos asentamientos. Esta mayor presencia de "tecnologías tradicionales" y menor representación de actividades recolectoras de recursos malacológicos seguirá vigente también durante el Neolítico en este área respecto a la zona occidental de la región cantábrica.

Con el objetivo de responder a la escasez de utillaje en algunos contextos, planteamos como hipótesis la utilización de las conchas de molusco, junto a otros soportes, como instrumentos para la realización de algunas actividades productivas de estos grupos. Esta hipótesis deriva tanto de estudios que confirman la utilización de tecno- logías confeccionadas sobre concha de molusco por parte de grupos de cazadores recolectores en otras áreas geográficas, como de la reiterada presencia de este recurso en los asentamientos litorales cantábricos. La combinación de ambos factores nos puso en alerta acerca del uso tecnológico que, una vez consumido el molusco, podría haber tenido su concha entre los grupos que llevaron a cabo la recolección. Esta hipótesis se ve apoyada además, por las diferentes experimentaciones exploratorias realizadas, en las cuales hemos comprobado la gran potencialidad de las conchas para trabajar sobre materias como la madera o la piel.

En este artículo se presentan los resultados del análisis funcional de nueve fragmentos de concha procedentes de los niveles neolíticos de la cueva de Santimamiñe (Bizkaia, País Vasco) a partir de los cuales se ha puesto a prueba la hipótesis propuesta. Para confirmar la utilización de estos restos en actividades productivas mediante un programa experimental se han comparado e interpretado las huellas de uso. A partir de los resultados obtenidos, se discuten aspectos metodológi$\cos$, las investigaciones relativas a la utilización de conchas como instrumentos en diversos contextos internacionales, y el eventual papel de instrumentos análogos en las sociedades mesolíticas y neolíticas de la región cantábrica.

\section{MATERIAL Y MÉTODOS}

El material arqueológico analizado fue seleccionado durante el estudio arqueomalacológico del yacimiento de Santimamiñe (Fig. 1), tras el hallazgo de una charnela de Ostrea edulis con morfología similar a la de algunos útiles líticos (Lám. I, n. ${ }^{\circ}$ 1). Esto nos llevó a revisar todo el material y a seleccionar todos aquellos fragmentos susceptibles de haber servido como instrumento de trabajo.

La revisión estratigráfica del yacimiento, emprendida entre los años 2004 y 2006 (López Quintana y Guenaga 2006-2007), ha puesto en evidencia nuevos niveles donde los moluscos aparecen de forma desigual: escasos restos en los niveles MSF (1) y Aziliense y considerables acumulaciones en los niveles mesolíticos y neolíticos. Los nueve fragmentos de conchas de molusco analizados proceden de los niveles neolíticos.

(1) Magdaleniense Superior Final. 

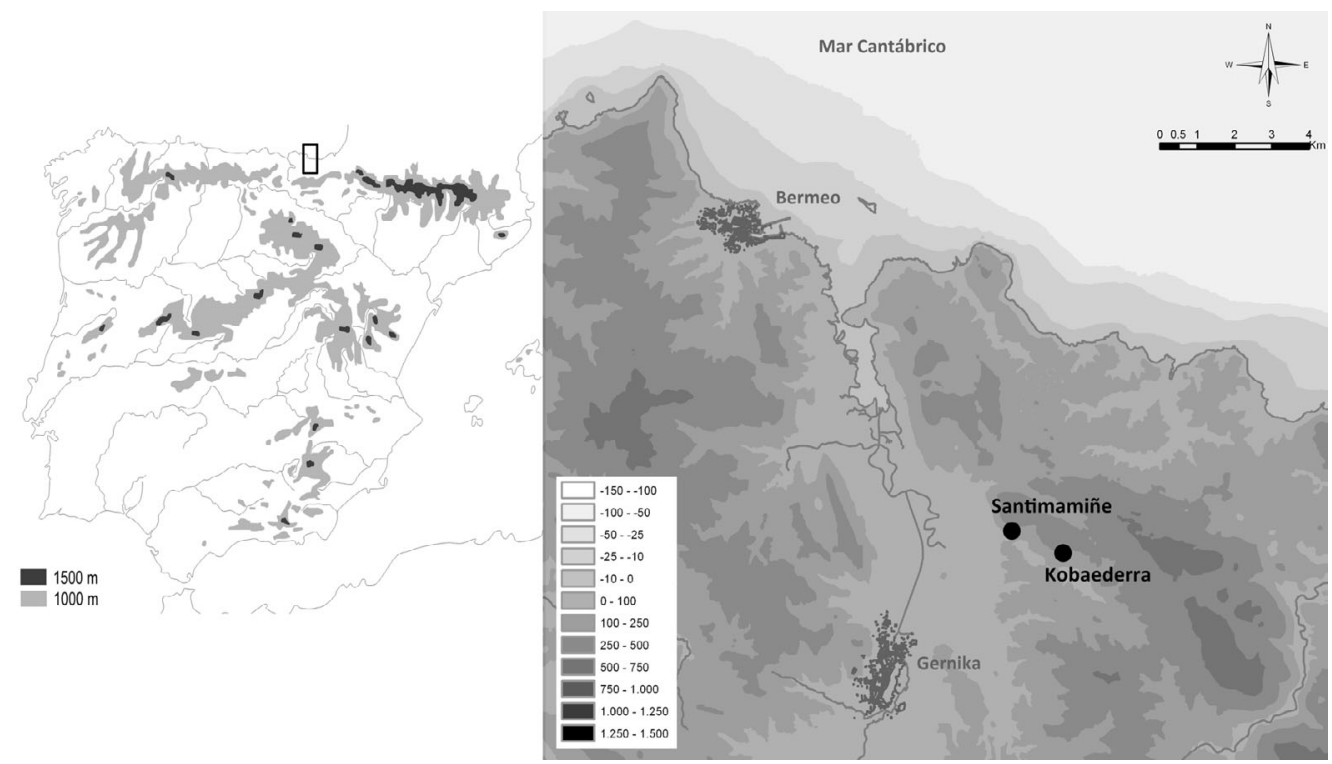

Fig. 1. Localización de la cueva de Santimamiñe en la zona de Urdaibai (Bizkaia).

Nivel Lsm (2): cuatro son de la especie Ostrea edulis (Linné 1758): dos de charnela de valvas derechas, uno de impresión de músculo aductor de valva derecha y otro de borde. Otros dos son un borde de Patella sp. y otro de Ruditapes decussatus (Linné 1758). Nivel Slm (3) se han analizado una charnela de valva derecha, una impresión de músculo aductor de valva derecha de Ostrea edulis, y otro fragmento de Mytilus galloprovincialis (Lamarck 1819). Siete de estos nueve fragmentos de concha, fueron definidos como instrumentos de trabajo (Lám. I) tras el análisis funcional (4).

Para contrastar la hipótesis inicial planteada a partir del mismo se han caracterizado mediante

(2) Denominación del nivel Lsm por la aplicación de criterios de estratigrafía analítica empleados en la reciente intervención efectuada en el yacimiento de Santimamiñe.

(3) Denominación del nivel Slm por la aplicación de criterios de estratigrafía analítica empleados en la reciente intervención efectuada en el yacimiento de Santimamiñe.

(4) Gutiérrez Zugasti, F.I.; Cuenca Solana, D.; Clemente Conte, I.; González Sainz, C. y López Quintana, J. (e.p.). "Instrumentos de trabajo y elementos de adorno en conchas de molusco de la cueva de Santimamiñe (Kortezubi, Bizkaia). En J. López Quintana (ed.): Excavaciones en la cueva de Santimamiñe. Campañas 2004-2006. Gobierno Vasco. Bilbao.

Cuenca Solana, D. 2009. Las "tecnologías invisibles" en los grupos de cazadores recolectores del litoral durante los inicios del Holoceno (9.500-5.000 uncal BP) en la región cantábrica. Utilización de las conchas de molusco en la realización de actividades productivas. Trabajo de Investigación inédito. Universidad de Cantabria. un programa experimental las huellas de uso generadas sobre los instrumentos de concha al llevar a cabo algunas actividades productivas. $\mathrm{Su}$

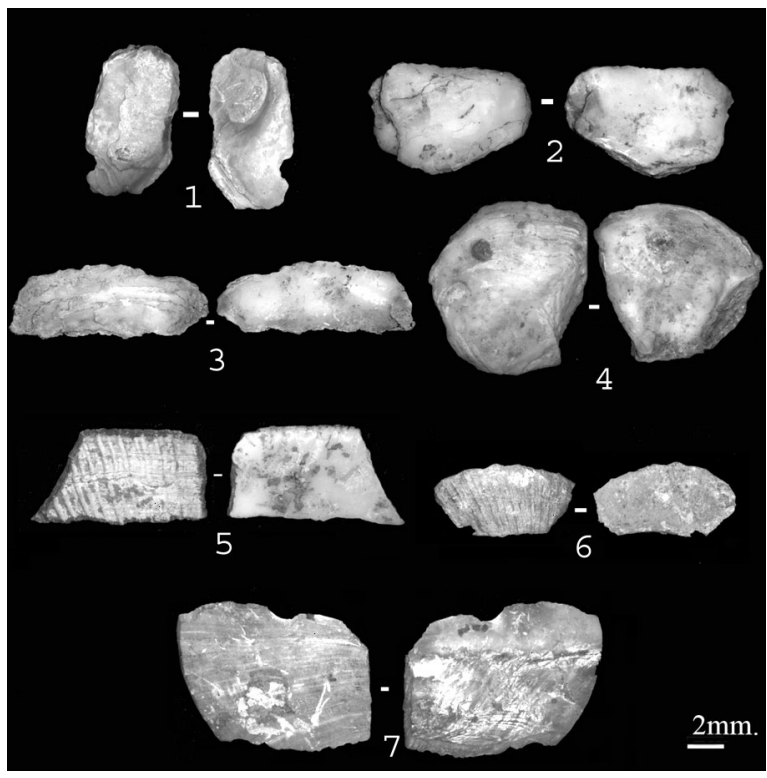

Lám. I. Instrumentos arqueológicos de concha documentados en los niveles de Neolítico inicial (Lsm-Slm) de Santimamiñe. 1) Charnela de Ostrea edulis. 2) Impresión de músculo aductor de Ostrea edulis. 3) Borde de Ostrea edulis. 4) Impresión de músculo aductor de Ostrea edulis. 5) Borde de Ruditapes decussatus. 6) Borde de Patella sp. 7) Borde de Mytilus galloprovincialis. Escala $2 \mathrm{~mm}$ a $5 \mathrm{X}$. 
objeto es la descripción del tipo de rastros de uso generados en los instrumentos, así como, el posterior análisis de los procesos que los conforman. Al mismo tiempo, se han controlado variables no modificables (como la materia trabajada) y modificables (la acción de trabajo, la duración del mismo y el ángulo de trabajo) que consideramos significativas. Esto garantiza una experimentación controlada o analítica (González e Ibáñez 1994: 17).

Las actividades seleccionadas, el procesamiento de madera, piel, fibras vegetales y cuero, son coherentes con los contextos arqueológicos definidos por el marco geográfico y cronológico propuesto (Clemente 1997; Gibaja 1993). Su elección deriva directamente de las hipótesis planteadas a partir de los resultados del análisis funcional de los instrumentos de trabajo procedentes del yacimiento de Santimamiñe (Kortezubi, Bizkaia) (5). Las especies de moluscos seleccionadas se corresponden con las analizadas en el material arqueológico. Como, a su vez están presentes en diferentes proporciones en los inventarios arqueomalacológicos de los yacimientos del marco cantábrico durante el Mesolítico y Neolítico inicial (6), su selección para la experimentación también es coherente y oportuna.

La observación macroscópica del material entre 5 y $72.5 X$ se hizo con una lupa binocular Leica MZ16A. Su objetivo era analizar y fotografiar macro-rastros resultantes del uso: principalmente redondeamientos y melladuras. Posteriormente las piezas han sido observadas y fotografiadas de nuevo entre 100 y $200 \mathrm{X}$ con un microscopio Leica DM2500M con el fin de observar y analizar los micro-rastros. Las superficies de los micropulidos y el fondo de las estrías han sido registrados utilizando un duplicador de Leica insertado en el propio microscopio, lo que permitía la observación y fotografía de estas superficies hasta 400X. Además, se ha manejado una lupa binocular Leica S8APO y un microscopio Leica DMLM fotografíando mediante cámara digital Canon EOS 450D con adaptador DSLRCC (LM Digital SLR Adapter with Canon Bayonet with C-Mount). Por último, para el montaje multifoco hemos utilizado el programa Helicon Focus versión 4.62.

(5) Gutiérrez Zugasti et al. (e.p.). Véase nota 4.

(6) Gutiérrez Zugasti, F.I. 2008. La explotación de moluscos y otros recursos litorales en la Región Cantábrica durante el Pleistoceno Final y Holoceno Inicial. Tesis Doctoral inédita. Universidad de Cantabria.
Las conchas escogidas para la experimentación se han limpiado con agua para su posterior observación y fotografía, eliminando, en su caso, otras partículas o residuos adheridos repasando las zonas activas mediante un bastoncillo de algodón impregnado de alcohol o de nafta.

\section{RESULTADOS DEL ANÁLISIS DE HUELLAS DE USO SOBRE EL MATERIAL MALACOLÓGICO DE SANTIMAMIÑE}

El análisis puso de manifiesto el empleo de siete conchas como instrumento en el procesado de materias de dureza blanda-media de origen animal y en algún caso también vegetal (7). Concretamente, se identificaron en todos los instrumentos acciones transversales de raspado, en algunos casos intercalando cinemáticas longitudinales.

Tres fragmentos de Ostrea edulis (Lám. I: 2, 3 y 4) presentaban rastros sobre uno de los bordes naturales (Lám. II: 1-4) y un cuarto huellas de uso en el vértice de la charnela de acusado redondeamiento (Lám. I: 1). Esta charnela mostraba un pulido caracterizado por una trama cerrada, brillo mate y aspecto graso.

Sendos fragmentos de Patella sp. y Mytilus galloprovincialis (Lám. I: 6 y 7) evidenciaban huellas de acción transversal en uno y dos de sus bordes respectivamente, provocadas al trabajar alguna materia animal de carácter abrasivo. Por último, un fragmento de Ruditapes decussatus con uno de sus bordes fuertemente redondeado, presentaba un pulido de trama cerrada-compacta, con estrías de fondo oscuro en oblicuo y en perpendicular al filo (Láms. I: 5 y III: 1-4). Este fragmento se relacionó con el procesado de algún vegetal rico en sílice.

\section{EL PROGRAMA EXPERIMENTAL}

Las conchas seleccionadas para el programa experimental corresponden a cuatro especies diferentes de moluscos. No se las ha sometido a ningún proceso de producción orientado a la formatización del instrumento, salvo a las conchas

(7) Gutiérrez Zugasti et al. (e.p.). Véase nota 4. 


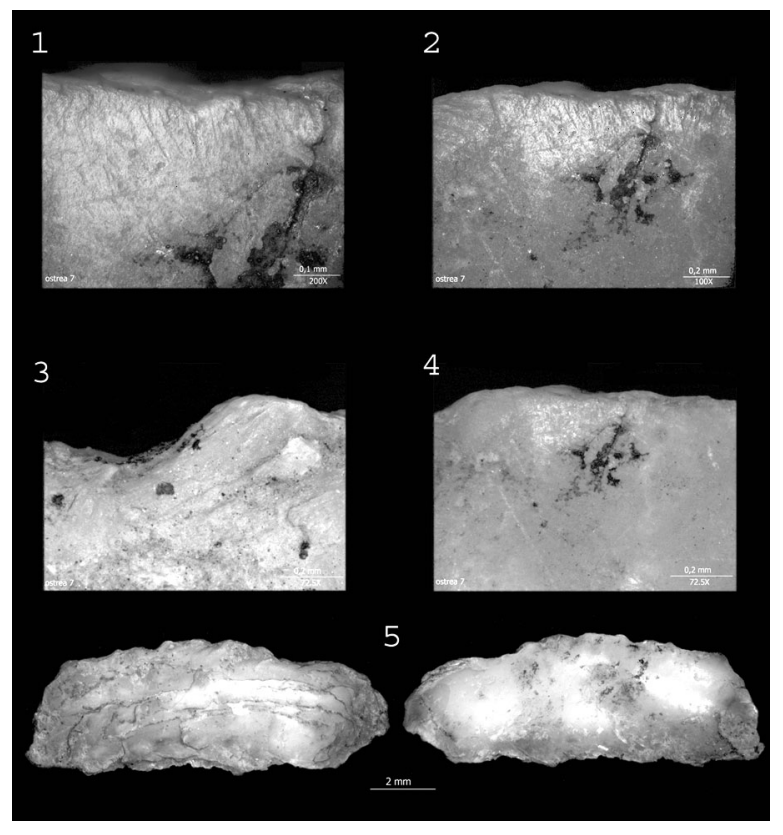

Lám. II. Huellas de uso evidenciadas en un fragmento de borde de Ostrea edulis procedente del nivel Neolítico Lsm de Santimamiñe (instrumento 3). 1) Escala 0,1 mm a 200X. 2) Escala 0,2 mm a $100 \mathrm{X}$. 3) Escala $0,2 \mathrm{~mm}$ a 72.5 X. 4) Escala $0,2 \mathrm{~mm}$ a 72.5 X. 5) Escala $2 \mathrm{~mm}$ a $5 X$.

de Ruditapes decussatus, las cuales han sido percutidas, para obtener aristas más adecuadas para trabajo de corte que el propio filo natural. Los demás, por tanto, son "instrumentos expeditivos" o basados en la utilización directa de los restos del propio animal (en este caso su concha) sin ninguna modificación (Binford 1998: 42).

Antes del programa experimental se han documentado los rastros tafonómicos y las alteraciones más genéricas que suele mostrar la concha de cada especie, a través de la observación y la fotografía de individuos recogidos en las mismas circunstancias pero no utilizados (Lám. IV). Este aspecto está orientado a diferenciar en las conchas las alteraciones naturales de aquéllas producidas por su utilización como instrumento.

En los bivalvos de morfología asimétrica como Ostrea edulis hemos experimentado con ambas valvas para intentar observar diferencias en la potencialidad del instrumento en cada caso, ya que sus diferencias de espesor, morfología y tamaño son evidentes.

La especie de molusco empleada es una variable significativa ya que, si bien todas las conchas comparten la composición mineralógica, en cada caso varía la proporción entre los minerales y su

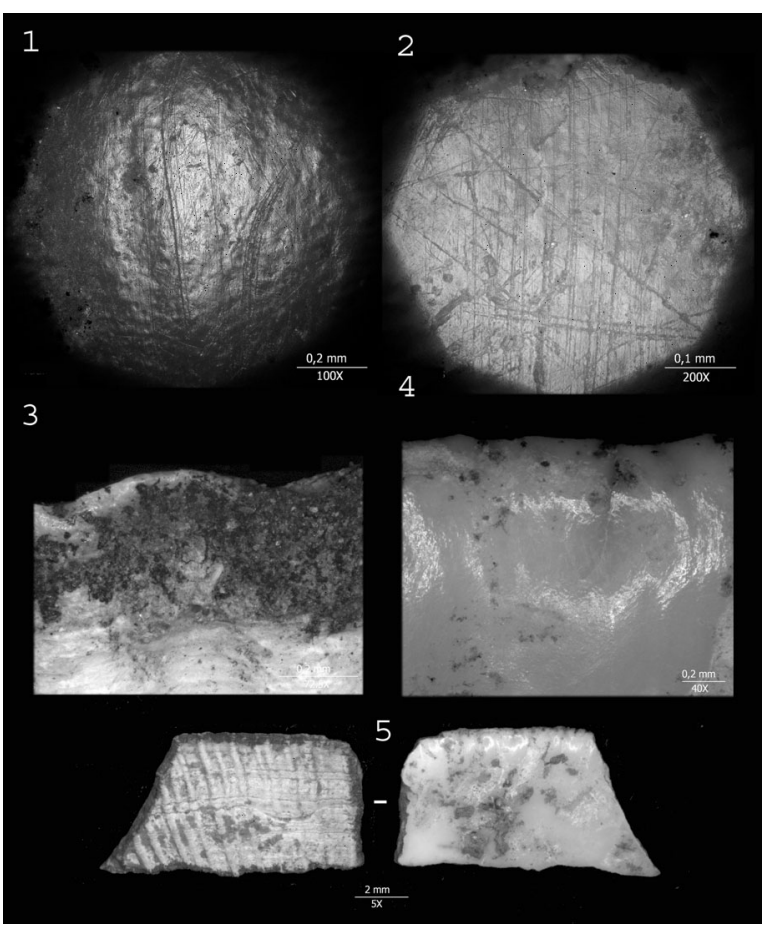

Lám. III. Huellas de uso evidenciadas en un fragmento de Ruditapes decussatus procedente del nivel Neolítico Lsm de Santimamiñe (instrumento 5). 1) Escala 0,2 mm a 100X. 2) Escala $0,1 \mathrm{~mm}$ a $200 \mathrm{X}$. 3) Escala $0,2 \mathrm{~mm}$ a $72.5 X .4)$ Escala $0,2 \mathrm{~mm}$ a $40 X .5)$ Escala $2 \mathrm{~mm}$ a $5 X$.

conformación microestructural, es decir, cristalización y disposición. Estas divergencias pueden provocar cualidades específicas ante la formación
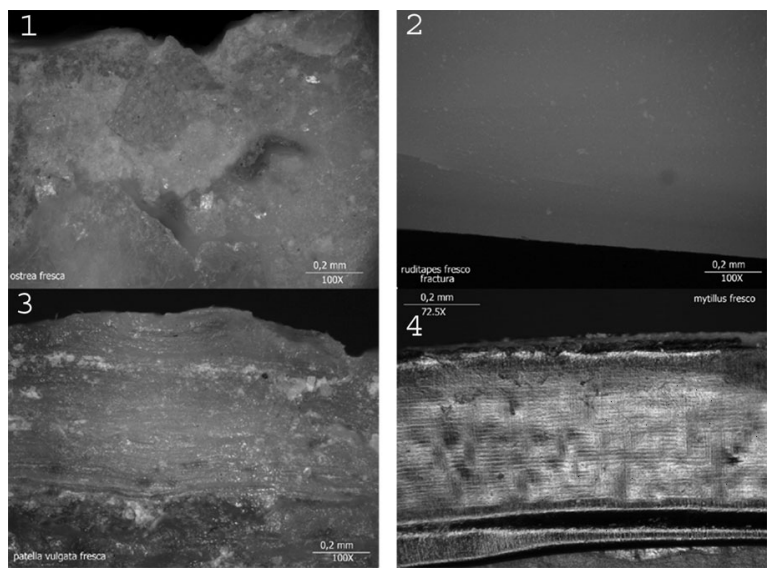

Lám. IV. Superficies de conchas recogidas en la playa sin alteraciones producidas por el uso. 1) Ostrea edulis. a 100X. 2) Ruditapes decussatus a 100X. 3) Patella sp. a $100 X .4)$ Mytilus galloprovincialis a 72.5X. Todas a escala $0,2 \mathrm{~mm}$. 
de las huellas de uso. Esta formación diferencial de los rastros puede afectar las diversas capas que conforman una misma concha, ya que su respectiva composición y estructuración también varía. En este sentido, las diferencias de coloración existentes entre cada una (periostracum, ostracum e hipostracum) suponen una dificultad desigual para la documentación de los rastros.

La materia trabajada con el instrumento es también una variable significativa y no modificable, a pesar de que el estado del material trabajado con el instrumento puede variar según se trate de materia humedecida, seca o de otros tipos (Clemente 1997: 29).

Para emprender el programa experimental se han seleccionado recursos vegetales (plantas no leñosas y madera) y animales (piel seca y fresca): varas de avellano en estado fresco (Corylus avellana, Linné 1753), tallo de junco fresco (Juncus sp.), piel fresca de cordero (Ovis aries Linné 1758), piel seca de corzo (Capreolus capreolus Linné 1758) y tiras de cuero de ciervo (Cervus elaphus Linné 1758).

Debido a la cinemática de las huellas registradas en los instrumentos de trabajo documentados en el yacimiento de Santimamiñe (8), así como a la escasa potencialidad funcional de las conchas en actividades de corte, al menos sin modificar sus características naturales, prácticamente todas las acciones realizadas en el programa experimental han sido transversales. Concretamente, consistieron en: acción transversal bidireccional de raspado con conchas de Ostrea edulis, Mytilus galloprovincialis y Patella sp. con el objetivo de limpiar materia grasa y restos cárnicos y adelgazar piel fresca de Ovis aries y seca de Capreolus capreolus; acción transversal bidireccional con charnela de Ostrea edulis para adelgazar y ablandar tiras de cuero de Cervus elaphus; acción transversal unidireccional de raspado con borde natural de Ruditapes decussatus para extraer fibras vegetales de Juncus sp., así como raspado en acción transversal unidireccional para extraer la piel y regularizar varas de Corylus avellana.

Con acciones de cinemática longitudinal, solamente se ha experimentado para el corte de fibras vegetales de Juncus sp. utilizando la arista obtenida por la fractura mediante percusión de una concha de Ruditapes decussatus.

(8) Gutiérrez Zugasti et al. (e.p.). Véase nota 4.
La fuerza en todos los casos se ha aplicado por presión con el instrumento sobre la materia trabajada.

El tiempo de utilización del instrumento es otra importante variable que influye decisivamente en la formación de los rastros de uso en los instrumentos de trabajo (Clemente 1997: 34; González e Ibáñez 1994: 31). En los instrumentos de concha, la formación de las huellas de uso es similar a la del utillaje lítico, pero éstas se producen más rápidamente debido a su composición, principalmente de aragonito (9). La utilización de este tipo de instrumentos respecto a los constituidos sobre soportes líticos se rentabiliza con la inmediatez de su uso expeditivo, y su abandono y sustitución por otro al decaer su efectividad, más aún en contextos donde las conchas son muy numerosas. Por ello, en esta primera aproximación experimental la duración de las actividades ha sido más breve que en los programas experimentales realizados con utillaje lítico.

Todos los experimentos se han realizado en tres intervalos temporales de 5, 10 y 15 minutos. Para cada intervalo se ha utilizado una concha, con el fin de repetir la observación directa con el microscopio sobre el instrumento una vez finalizado el experimento. Buscamos así, comprender mejor la evolución de los rastros de uso en cada caso.

El ángulo que conforma el instrumento con la materia trabajada es también significativo en la formación de los rastros de uso (Clemente 1997: 33). A pesar de la dificultad de controlar las variaciones leves durante la realización mecánica del experimento, a veces se ha intentado modificar la inclinación del útil para registrar la ubicación de las huellas de uso en la cara externa e interna de la concha en cada caso. Casi todos los ángulos de contacto han sido rectos (en torno a $90^{\circ}$ ), tanto en las acciones transversales, como longitudinales, pero, se ha variado en ocasiones entre los $75^{\circ}$ y los $90^{\circ}$ Los únicos experimentos en los que el ángulo no tiende a ser recto corresponden al tratamiento de cuero con la charnela de

(9) Clemente, I. 1995. Instrumentos de trabajo líticos de los Yámanas (Canoeros-nómadas de la Tierra del Fuego): una perspectiva desde el análisis funcional. Tesis Doctoral inédita. Departament d'Antropologia Social i Prehistoria, UAB, Barcelona.

Mansur, M. y Clemente, I. (e.p.). “¿Tecnologías invisibles? Confección, uso y conservación de instrumentos de valva en Tierra del Fuego". XIV Congreso Nacional de Arqueología Argentina (Rosario, 2001), Argentina. 
Ostrea edulis: la fricción de esta zona de la concha con la piel forma un ángulo prácticamente plano $\left(180^{\circ}\right)$, ya que la superficie de la charnela es plana.

\section{RESULTADOS DEL PROGRAMA EXPERIMENTAL}

\subsection{Madera}

Al tratarse de una cinemática de raspado unidireccional el pulido se ubica en la cara interna de la concha de Ruditapes decussatus. Este pulido presenta un aspecto brillante y plano, la trama es compacta y la microtopografía lisa (Lám. V: 1 y 3). A su vez el filo muestra un redondeamiento de grado alto tras 15 minutos de trabajo con el instrumento. Los rastros microscópicos de los instrumentos son las estrías de fondo oscuro, finas y largas perpendiculares al filo, junto a otras más finas, casi como arañazos, orientadas en oblicuo al filo (Lám. V: 3).

El rasgo más destacable del proceso es la pérdida de la capa superficial de la concha o periostracum, cuyo aspecto, es similar al "descascarillado" de las zonas que han estado en mayor contacto con la materia trabajada (Lám. V: 2).

\subsection{Planta no leñosa}

Se ha llevado a cabo una acción de raspado, que localiza el pulido principalmente en la cara interna del borde de la concha de Ruditapes decussatus, en la zona de la arista del filo y, en menor medida, en la cara externa al perder la capa rugosa del periostracum.

El pulido presenta una trama cerrada y la microtopografía es lisa en la zona del filo. Hacia el interior de la concha la trama se abre convirtiéndose en semicerrada (Lám. VI: 4). Ésta es cerrada, brillante y de microtopografía rugosa en la propia arista del borde de la concha.

En la cara externa el pulido es menos brillante casi mate, de trama cerrada y microtopografía rugosa. El redondeamiento del filo es más acusado en la cara interna de la concha pero, en todo caso, de grado bajo-medio.

Los instrumentos que han procesado planta no leñosa presentan pequeñas melladuras, principal-
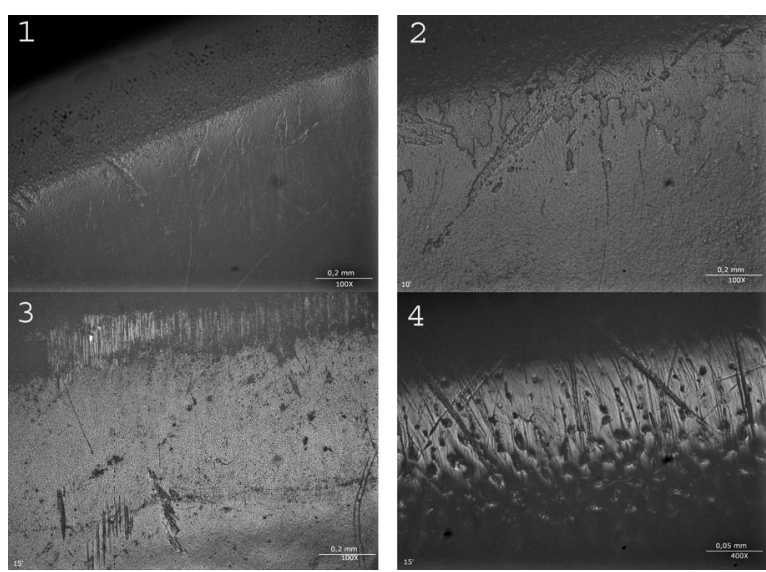

Lám. V. Huellas de uso documentadas en los instrumentos experimentales de Ruditapes decussatus utilizados para procesar madera. 1) Pulido en la zona del borde en la cara interna tras 10 minutos de trabajo. Escala $0,2 \mathrm{~mm}$ a 100X. 2) "Descascarillado" producido en la capa superficial de la cara interna. Escala 0,2 mm a 100X. 3) Pulido en la cara interna tras 15 minutos de trabajo. Escala $0,2 \mathrm{~mm}$ a 100X. 4) Huellas de uso sobre la zona del borde tras 15 minutos. Escala $0,05 \mathrm{~mm}$ a $400 \mathrm{X}$.

mente en forma de media luna, o semicirculares que progresivamente tienden a alinearse.

En las zonas redondeadas del filo se forman estrías compuestas, cortas y de fondo brillante (Lám. VI: 2 y 3). Hacia el interior de la concha
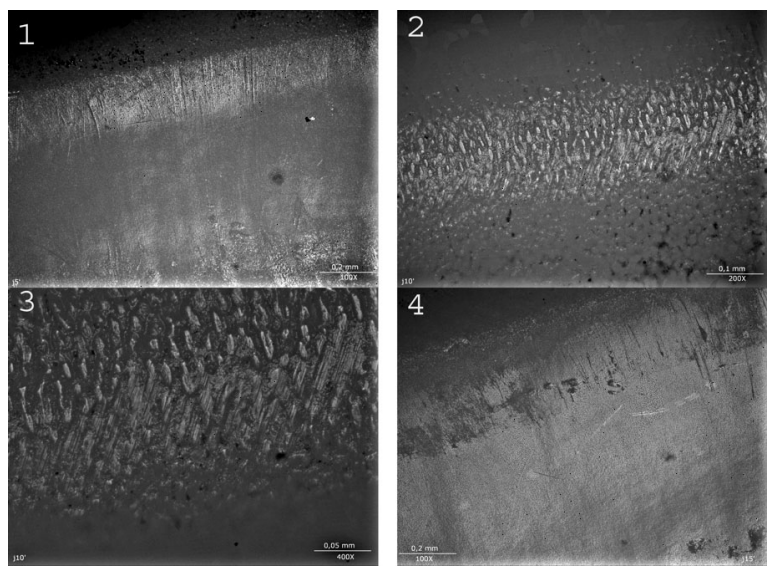

Lám. VI. Huellas de uso documentadas en los instrumentos experimentales de Ruditapes decussatus utilizados para procesar planta no leñosa. 1) Pulido en la zona del borde en la cara interna tras 5 minutos de trabajo. Escala $0,2 \mathrm{~mm}$ a $100 \mathrm{X}$. 2) Alteración en la zona del borde de la cara interna tras 10 minutos. Escala $0,1 \mathrm{~mm}$ a $200 \mathrm{X}$. 3) Detalle de la zona anterior. Escala $0,05 \mathrm{~mm}$ a $400 \mathrm{X}$. 4) Huellas de uso sobre la zona del borde de la cara interna tras 15 minutos. Escala 0,2 mm a $100 \mathrm{X}$. 
las estrías son más largas, finas y de fondo oscuro, distribuidas longitudinalmente y en los instrumentos utilizados en acciones longitudinales de corte también en oblicuo.

Sobre la arista las estrías son menos numerosas también finas y muy largas dispuestas longitudinalmente (Lám. VI: 1). En la cara externa de la concha las estrías son de fondo oscuro, finas y largas, dispuestas en sentido longitudinal al filo.

\subsection{Piel fresca}

En las conchas empleadas para procesar piel en estado fresco a partir de los 10 minutos de trabajo comienza a desarrollarse un pulido en la cara interna, localizado marginalmente en el borde de la concha. En 15 minutos se desarrolla en la cara externa y sobre la arista redondeada.

El pulido tiene una trama cerrada, es brillante y tiene un aspecto muy graso. La microtopografía es ligeramente rugosa, tendiendo a alisarse en las zonas más elevadas de la concha (Lám. VII: 3 y 4). En las conchas de Mytilus la trama del pulido llega a ser compacta en las zonas más elevadas de la topografía de la cara interna.

En las conchas de Mytillus galloprovincialis se aprecia un desarrollo más rápido del pulido en la cara interna respecto a Ostrea edulis y a Patella sp. (Lám. VII: 1). A partir de los 15 minutos se empieza a apreciar cierto desarrollo del pulido en el borde de la capa externa. Es de trama cerrada, brillante y de aspecto graso, con microtopografía rugosa. El redondeamiento del filo llega a un alto grado tras 15 minutos de trabajo.

En las conchas de Ostrea edulis se aprecia la fractura de las capas rugosas de la cara externa. Tiende a regularizarse la superficie de círculos regulares continuos que conforma la cara externa en esta especie.

Tanto en las conchas de Ostrea edulis como en las de Patella sp sobre el borde de la capa rugosa de la cara externa se desarrollan algunas melladuras de morfología semicircular aisladas.

En 15 minutos aparecen estrías en la cara interna, más profundas y anchas en perpendicular al filo, y otras oblicuas más superficiales y finas Ambas son irregulares y de fondo oscuro (Lám. VII: 2).

En los instrumentos de Mytilus galloprovincialis y Patella sp., pero no en los Ostrea edulis,
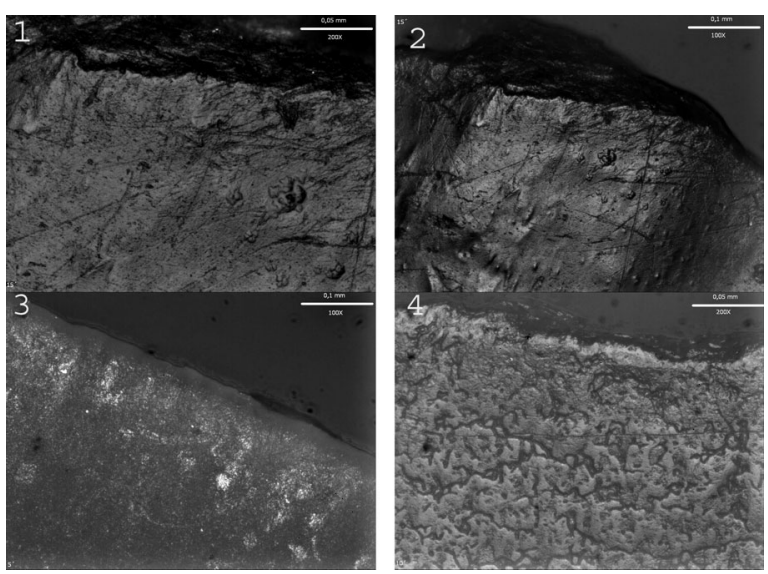

Lám. VII. Huellas de uso documentadas en los instrumentos experimentales utilizados para procesar piel fresca. 1) Pulido en la zona del borde en la cara interna de $\mathrm{Pa}$ tella sp. tras 15 minutos de trabajo. Escala $0,05 \mathrm{~mm}$ a 200X. 2) Borde de la cara interna de Patella sp. con pulido de brillo mate y estrías de fondo oscuro tras 15 minutos de trabajo. Escala 0,1 mm a 100X. 3) Inicio del desarrollo del pulido en la cara interna de Mytilus galloprovincialis tras 5 minutos de trabajo. Escala $0,1 \mathrm{~mm}$ a $100 \mathrm{X}$. 4) Huellas de uso sobre la zona del borde de Ostrea edulis tras 10 minutos. Escala $0,05 \mathrm{~mm}$ a $200 \mathrm{X}$.

aparecen de microagujeros irregulares y de fondo oscuro en la cara interna de la concha.

\subsection{Piel seca}

Se aprecia la pérdida del periostracum en algunas zonas de la cara interna en los instrumentos de Ostrea edulis, conformando diferentes alteraciones. Sobre el ostracum el pulido tiene un brillo mate, la trama es cerrada y la microtopografía rugosa, mientras que sobre las zonas que han permanecido del periostracum o capa más superficial, el pulido está menos desarrollado, la trama es semicerrada y la microtopografía rugosa (Lám. VIII: 1).

En las conchas de Mytilus el pulido se concentra principalmente sobre las elevaciones de la topografía ondulada, mientras que en las de Patella sp. la trama llega a ser compacta en esas zonas. En todas las conchas experimentales que han procesado piel en estado seco, en la cara externa, el pulido tiene la trama cerrada y un aspecto graso. El redondeamiento del filo llega a grado alto tras 15 minutos de trabajo. 

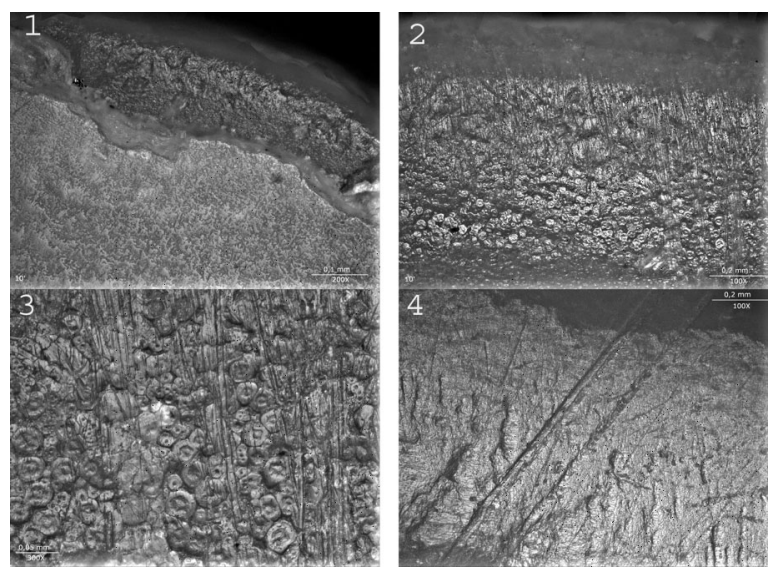

Lám. VIII. Huellas de uso documentadas en los instrumentos experimentales utilizados para procesar piel seca. 1) Fractura de la capa superficial en la zona del borde de Ostrea edulis y desigual desarrollo del pulido tras $10 \mathrm{mi}-$ nutos de trabajo. Escala 0,1 mm a 200X. 2) Huellas de uso en la cara externa de Ostrea edulis tras 10 minutos de trabajo. Escala 0,2 mm a 100X. 3) Detalle de la zona anterior, con regularización de la capa exterior de la concha y numerosas estrías de fondo oscuro. Escala $0,05 \mathrm{~mm}$ a $300 X$. 4) Detalle de estrías de fondo oscuro en la zona del borde de Ostrea edulis tras 15 minutos. Escala 0,2 $\mathrm{mm}$ a $100 \mathrm{X}$.

En las conchas de Ostrea edulis y Patella sp. se fractura la capa superior de la concha (periostracum) formando dos filos superpuestos. Por el contrario solamente los instrumentos de Ostrea edulis muestran, tras 15 minutos de trabajo, las huellas provocadas por la prensión con la mano del instrumento. Son visibles en la zona de la charnela en la cara externa y en la zona de la impresión del músculo aductor en la cara interna.

En los instrumentos de Mytilus, así como en los de Patella sp. se han registrado desconchados o mellamientos semicerrados con morfología semicircular o de media luna, localizados de forma aislada en el filo que tienden a alinearse progresivamente. En las conchas de Mytilus las melladuras abruptas del filo, en ocasiones, conforman un aspecto casi dentado.

En la cara interna se han registrado estrías de fondo oscuro, anchas, cortas y profundas, localizadas en la zona de la arista y orientadas en perpendicular al filo (Lám. VIII: 4). En la cara externa las estrías son muy numerosas, de fondo oscuro, muy finas y largas (Lám. VIII: 2 y 3). En los instrumentos de Mytilus y Patella sp. se han documentado microagujeros de morfología circular.

\section{COMPARACIÓN ENTRE LOS RESULTADOS DEL ANÁLISIS SOBRE EL MATERIAL MALACOLÓGICO DE SANTIMAMIÑE Y EL PROGRAMA EXPERIMENTAL}

Los resultados del programa experimental confirman las hipótesis planteadas respecto a las materias procesadas con los instrumentos de concha documentados en Santimamiñe. Concretamente, en lo que respecta al fragmento de Ruditapes decussatus, la experimentación parece apuntar más al procesado de alguna planta no leñosa que de la madera. Sin embargo, a pesar de la similitud entre las características de los rastros descritos a nivel arqueológico y experimental, se evidencia un mayor desarrollo de éstos en el material arqueológico analizado. En este sentido, el fragmento de Ruditapes decussatus procedente del nivel Lsm de Santimamiñe probablemente fue utilizado para procesar alguna planta no leñosa de mayor dureza que Juncus sp., o durante más tiempo que el empleado en los experimentos. Este último factor es muy probable, teniendo en cuenta que su duración máxima fue de 15 minutos, tras los cuales el instrumento aún conservaba una correcta potencialidad que podría haber permitido continuar el procesado de más materia vegetal.

En lo que se refiere a la charnela de Ostrea edulis con redondeamiento en el vértice, la experimentación realizada para ablandar tiras de cuero de pequeñas dimensiones, muestra rastros diagnósticos muy similares, quizás algo más desarrollados en las piezas experimentales. Entendemos que el estado más o menos seco de la piel puede ser un factor que debido al potencial de abrasión de esta materia provocara el desarrollo de rastros más marcados o de forma más rápida sobre la concha.

Los restantes fragmentos de concha procedentes de Santimamiñe, interpretados como instrumentos para raspar materia animal de dureza blanda-media, según la experimentación estarían más cercanos al procesado de piel en estado seco o a la utilización de algún abrasivo para curtir la piel (como el ocre, por ejemplo). Este uso está documentado etnográficamente de forma bastante habitual y provoca rastros más desarrollados y superficies más estriadas sobre los instrumentos (Lám. IX).

Esta hipótesis se basa en los rastros diagnósticos de ambas colecciones: el desarrollo del pu- 


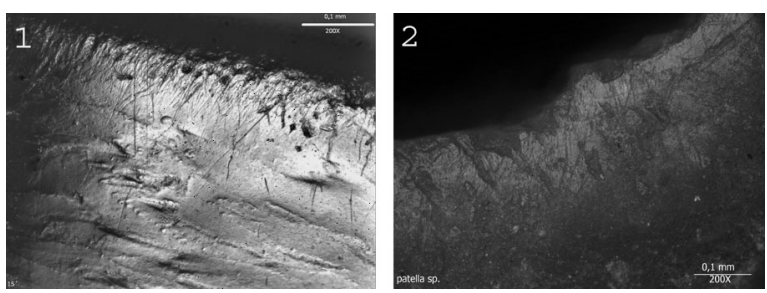

Lám. IX. Comparación de las huellas de uso entre el material arqueológico y experimental. 1) Borde de instrumento experimental de Patella sp. empleado para procesar piel seca a 200X. 2) Borde de Patella sp. procedente del nivel Neolítico inicial Lsm de Santimamiñe (instrumento 5) a 200X. Ambas a escala 0,1 mm.

lido, así como la concentración de estrías que presenta mayor similitud entre el material arqueológico y la muestra experimental que ha sido utilizada para trabajar piel en estado seco. La piel seca es bastante más abrasiva que la piel en estado fresco, generando un mayor desarrollo de los rastros y mayor presencia de estrías. En cualquier caso, consideramos que los rastros diagnósticos observados y documentados en el programa experimental permiten diferenciar al menos el tipo de materia procesada, así como el movimiento ejercido durante el trabajo. Probablemente, al aumentar la duración de los experimentos podremos conseguir rastros aún más diagnósticos. De la misma forma, al introducir en el futuro más variables modificables en la experimentación podremos incrementar los criterios para acercarse al material arqueológico con mayor precisión de análisis.

Por otra parte, observamos otra correlación significativa entre el material arqueológico analizado y los resultados obtenidos en el programa experimental. Los fragmentos de bivalvos procedentes de Santimamiñe analizados, se corresponden con diferentes partes anatómicas de valvas derechas. A través de la experimentación hemos comprobado cómo en el caso de las conchas de Ostrea edulis también cuentan con una mayor potencialidad funcional que las valvas izquierdas. Este aspecto deriva de la morfología más plana de las valvas derechas respecto a la forma más cóncava que presentan las izquierdas. Esta morfología plana parece dotar a su borde natural de una mayor resistencia ante la fractura al utilizar la concha como instrumento de trabajo.

Aunque por el momento tenemos poca información para poder confirmar este aspecto, parece significativo que la mayor potencialidad observa- da de forma experimental para las acciones de raspado en las valvas derechas de Ostrea edulis, coincida con la utilización de valvas derechas en todos lo casos analizados en el material arqueológico. A este respecto será necesario analizar más material malacológico procedente de contextos arqueológicos para intentar verificar con mayor seguridad esta observación.

\section{DISCUSIÓN Y CONCLUSIONES}

\subsection{Aspectos metodológicos}

Durante la realización del programa experimental y la posterior observación y caracterización de las huellas generadas en los instrumentos de concha, hemos podido documentar diferentes aspectos de carácter metodológico, tanto referentes al comportamiento de las conchas como instrumentos, como a la propia observación de las huellas formadas sobre éstos.

En primer lugar, la formación de rastros de uso sobre los instrumentos de trabajo es más rápida que en otras materias utilizadas para la manufactura de utillaje como el sílex. Hemos evidenciado la formación de rastros en trabajos de corta duración, incluso en 1 minuto (trabajo de madera con concha de Patella sp.). En comparación con las materias líticas, destaca la formación mucho más acusada de estrías. Durante la observación de la evolución de las huellas hemos podido verificar cómo, en ocasiones antes de que se desarrolle el pulido, ya se han formado estrías muy evidentes y a veces también bastante numerosas. Esto podría deberse a que se trata de superficies muy lisas, de forma similar a lo que ocurre con superficies como los cristales o la obsidiana. Del mismo modo, la fractura del borde de la concha y el posterior arrastre de estas pequeñas partículas desprendidas durante las acciones de trabajo podrían funcionar como un abrasivo que contribuyese de forma paralela a la formación de un elevado numero de estrías.

Consideramos de gran importancia para el futuro una mayor comprensión de la composición mineralógica y la diferente cristalización de las capas que conforman la concha, en relación con la formación de las huellas producidas por el uso. Sobre las superficies de las capas que componen la concha hemos detectado un diferente desarrollo de los rastros formados durante el trabajo con 
una misma materia. La topografía irregular, conformada por la pérdida de alguna de las capas superficiales de la concha, podría haber influido en la formación de rastros diferentes en cada zona, con un mayor o menor desarrollo de las huellas de uso debido al diferente grado de contacto con la materia trabajada de cada zona.

Sin embargo, también conocemos que varían en cada capa la composición mineralógica, el aspecto visual, así como la forma en que cristalizan los minerales constituyentes, lo cual también podría influir decisivamente en la formación de rastros. Del mismo modo, al comparar conchas de diversas especies usadas para trabajar una misma materia, hemos documentado en algunos casos alteraciones también diferentes. Esto podría deberse a la propia morfología de la concha, y por tanto al divergente contacto ejercido sobre la materia trabajada, en cierta medida también a la variación que existe en la composición entre cada especie y a la estructuración de los minerales que la conforman, o incluso a ambos aspectos.

La pérdida de la capa superficial o periostracum de la concha durante la utilización provoca la pérdida de los rastros de uso que se forman en esa superficie. A no ser que se siga utilizando el instrumento y formándose los rastros en la capa inferior o nacarada de la concha este aspecto podría generar un proceso de "invisibilidad arqueológica" que impediría la correcta caracterización de este material malacológico como instrumento de producción (10).

Como aspectos metodológicos relacionados con la observación y la documentación de rastros de uso en este tipo de instrumentos de concha, reseñamos la necesidad del uso de sistemas de observación macroscópicos (lupa binocular) y microscópicos (microscopio metalográfico). En los últimos años parece que el debate acerca de la conveniencia del uso de altos o bajos aumentos para la observación de las huellas de uso en instrumentos de producción líticos se ha ido cerrando, asumiéndose comúnmente entre la mayor parte de investigadores que ambos son compatibles, complementarios, e incluso necesarios. Sin embargo, este aspecto no se ha extendido de forma tan genérica al estudio de utillaje manufacturado con materias primas de origen animal, ya que existen numerosos trabajos basados tan sólo en la observación macroscópica (mayoritarios) o mi-

(10) Cuenca Solana, D. 2009. Véase nota 4. croscópica de los rastros. En lo que se refiere a la observación de las huellas de uso en instrumentos de concha, consideramos necesario el empleo de ambos medios de observación, ya que en cada caso responden a la observación específica de rastros difícilmente documentables empleando sólo altos o bajos aumentos. En este sentido, mientras la localización de las melladuras, redondeamientos o la primera documentación de las zonas activas del instrumento se ven favorecidas por el empleo de la lupa binocular, la observación y descripción del pulido y su microtopografía, así como la morfología de los microagujeros o las estrías obligan necesariamente a la utilización de al menos 200X, viéndose incluso favorecida su caracterización con el empleo de hasta 400X.

Otro aspecto muy importante relacionado con la observación específica de estos instrumentos es la importancia de la luz y la inclinación de la pieza. Un posicionamiento plano favorece la observación de los pulidos y las estrías, mientras que un posicionamiento vertical permite visualizar mejor las alteraciones de la arista y el filo, tales como el redondeamiento o las micro-melladuras. Este aspecto es especialmente característico en este tipo de utillaje, debido a la morfología más o menos cóncava, natural de las conchas.

El tratamiento muy cuidado de la luz se hace imprescindible, sobre todo en las conchas de Ostrea edulis o Ruditapes decussatus, ya que su aspecto muy nacarado, de tonos blancos muy brillantes, obliga al empleo de baja intensidad de luz para la observación y documentación fotográfica de los rastros de uso. Este aspecto, se agrava al emplear cámaras digitales con adaptador al microscopio, ya que capturan mucha luz al abrir el objetivo, obligando paralelamente a bajar la intensidad y a disminuir el tiempo de exposición durante la apertura del diafragma para obtener una buena imagen. Esto ocurre también al fotografiar otras materias como el hueso. En este sentido, el uso de réplicas de las superficies en papel de acetato puede ayudar a observar y registrar los micro-rastros en esas superficies tan brillantes (Banks y Kay 2003; D'Errico et al.1982; D'Errico 1988; Ilkjaer 1979; Knutsson y Hope 1984; Plisson 1983, 1984).

La vía de análisis abierta desde la aplicación de los estudios traceológicos a los instrumentos de concha requiere, sin duda, un esfuerzo orientado a mejorar y completar la metodología existente hasta ahora. Consideramos que el desa- 
rrollo metodológico orientado a buscar mejoras en el planteamiento de los programas experimentales, así como a la observación y descripción de las huellas de uso formadas sobre estos instrumentos de producción, puede contribuir a aumentar nuestro conocimiento acerca de las actividades económicas emprendidas por algunos grupos de cazadores recolectores. Paralelamente, el desarrollo metodológico de este tipo de analíticas debería llevar aparejada la modificación de la visión de los restos arqueomalacológicos exclusivamente como desperdicios del consumo bromatológico. Para ello se hace obligado un adecuado tratamiento de estas evidencias arqueológicas desde el propio trabajo de campo, así como su posterior procesado y almacenamiento, encaminado a permitir su ulterior estudio como instrumento.

\subsection{Las investigaciones sobre la utilización de conchas como instrumentos: una revisión crítica}

Hasta ahora son escasos los estudios arqueológicos que se han propuesto conocer las tecnologías confeccionadas con concha de molusco en las actividades productivas de los grupos humanos. En los últimos tiempos este campo de la Arqueología parece que ha visto aumentado de forma cuantitativa y cualitativa su alcance, gracias a estudios arqueomalacológicos y tecnológicos. El mayor auge de este tipo de análisis corresponde a áreas geográficas cuyos pobladores cuentan con una extensa herencia cultural del uso de las conchas, que incluso, en algunos casos, ha llegado hasta nuestros días. La zona del Pacífico (Choi y Driwantoro 2007; Jones y Keegan 2001; Szabó 2008; Szabó et al. 2007, entre otros) o Sudamérica (Bonomo 2007; Bonomo y Aguirre 2009; Dacal Moure 1978, entre otros) han sido las áreas donde algunos/as investigadores/ras vinculados a la arqueología han mostrado mayor interés por estudiar las conchas, como recurso alimenticio y adorno, y también como materia prima orientada a la manufactura de instrumentos de producción. Con mucha probabilidad, este desarrollo derive en gran medida de la existencia en estas latitudes de especies muy apropiadas de bivalvos y gasterópodos de gran tamaño, muy robustos, y susceptible de utilización cada una de sus partes anatómicas (columela, labio, espiras...) con fines diferenciados y específicos.
Estos estudios han tenido cierto eco en Europa, con algunos trabajos pioneros de Taborin (Taborin 1974) en los años 70, y desde mediados de los años 80 las aportaciones de Vigié y Courtin (1986; 1987; Vigié 1987; Vigié 1995, entre otros), centradas en yacimientos mesolíticos y neolíticos de la costa atlántica francesa.

Actualmente hay investigaciones a este respecto, en Italia (Cristiani et al. 2005), Grecia (Karali 2005) y también últimamente en España (11) (Maicas Ramos 2008; Pascual Benito 2008; Rodríguez y Navarro 1999). Algunas de estas aportaciones presentan deficiencias, en ocasiones similares a las que, según nuestra perspectiva, se plantean en los estudios tecnológicos aplicados sobre otras materias como el sílex. En este sentido, muchas de estas investigaciones acerca del utillaje de concha, se basan en criterios tipológicos para la clasificación de estos artefactos, la asignación de la funcionalidad desde la morfología, o el uso de experimentaciones que apenas llegan a ser de carácter exploratorio. Otras aproximaciones, no obstante, se orientan hacia la aplicación de la única metodología que, bajo nuestra perspectiva, puede definir realmente la función de un instrumento de producción: el análisis funcional (Semenov 1981). Entre estas investigaciones existe, de igual modo, una gran heterogeneidad.

Por un lado, es poco común la exposición de la metodología empleada en el análisis, y aún menor, la necesaria documentación gráfica de las huellas de uso reconocidas en los instrumentos. En otros casos, cuando la descripción de la metodología existe, la formulación de conclusiones se basa tan sólo en la observación de las alteraciones macroscópicas. Estas evidencias, si bien pueden ser válidas para diferenciar un instrumento de una concha que no ha sido utilizada, son insuficientes para analizar el tipo de actividad que se ha llevado a cabo, así como la materia trabajada.

Estos defectos metodológicos dificultan en gran medida la cohesión y fortalecimiento de unas bases sobre las que asentar la aplicación del método de análisis funcional a los instrumentos de producción de concha. Por el contrario, los trabajos basados en el análisis funcional aplicado a estas tecnologías (Cristiani et al. 2005; Lammers-Keijsers 2008; Rodríguez y Navarro 1999; Toht y Woods 1989; entre otros) confirman la

(11) Véase nota 4. 
gran aportación que pueden realizar este tipo de aproximaciones al conocimiento de las actividades productivas desarrolladas por los grupos humanos que han explotado el área litoral.

\subsection{Utilización de conchas como instrumentos en la región cantábrica}

Los siete fragmentos de material malacológico procedente de Santimamiñe, utilizados como instrumento de trabajo en diferentes actividades productivas son la primera evidencia de este tipo para contextos de la región cantábrica. Los resultados del análisis efectuado sobre el material arqueomalacológico y su comparación con el procedente del programa experimental refuerzan la hipótesis planteada inicialmente, es decir la utilización de conchas de molusco como instrumento en diversas actividades productivas por parte de los grupos del Mesolítico y Neolítico inicial en algunos contextos litorales. Sin embargo, el reducido numero de piezas analizadas, y sobre todo la imposibilidad de, hasta el momento, poner en relación estos datos junto con análisis similares del resto de utillaje (lítico y óseo) dificulta extraer conclusiones de carácter más amplio. Consideramos que ampliando este tipo de análisis a nuevos conjuntos malacológicos y poniendo estos datos en relación con estudios traceológicos aplicados sobre el utillaje lítico y óseo, pendientes aún en Santimamiñe, podríamos estar en disposición de valorar de forma más concluyente el papel de las conchas como instrumento dentro del abanico tecnológico de estos grupos. Mediante esta vía podremos valorar la utilización tecnológica de las conchas de molusco, frente al empleo de otros soportes. Igualmente podremos analizar si esta utilización guarda alguna relación directa con actividades productivas específicas o si, por el contrario, la inmediatez que supone el empleo de un recurso natural muy numeroso, y aportado previamente al yacimiento con una finalidad bromatológica, pudo suponer un aliciente para su uso tecnológico.

A pesar de que la reducida extensión del análisis realizado dificulta extraer conclusiones de carácter socio-económico parece probable que los factores y circunstancias indicadas, pudieran hacer disminuir en cierta medida el esfuerzo orientado a la captación de otras materias primas, así como a su posterior proceso de producción o for- matización, si es que las conchas fueron usadas sin transformación. Sin duda, este aspecto podría modificar ciertas conductas de comportamiento de estos grupos en relación a los procesos de manufactura del utillaje. Y por extensión al resto de procesos de producción, del mismo modo en que probablemente éstos pudieron verse influidos también por aspectos como la disponibilidad dentro del área local de materias primas de calidad, la necesidad de llevar a cabo desplazamientos a grandes distancias o de desarrollar una estrecha red de relaciones sociales con el fin de disponer de los recursos minerales necesarios para manufacturar sus instrumentos de trabajo (12).

Por otra parte, si se tiene en cuenta que las evidencias documentadas hasta el momento se corresponden con un área, la zona oriental de la región cantábrica, con una mayor disponibilidad de materias primas líticas de buena calidad para la talla respecto a los contextos de la parte occidental, se refuerza aún más el papel que los instrumentos en concha pudieron haber jugado en el desarrollo de las actividades productivas. En este sentido, cabe esperar que la posibilidad de éxito en el registro de nuevas evidencias de la utilización instrumental de las conchas sea aún mayor para contextos con un menor índice de "tecnologías tradicionales", como los concheros asturienses de la zona occidental de la región cantábrica. En el futuro la documentación de tecnología de concha en contextos asturienses podría contribuir a paliar la escasez de utillaje sobre la que tradicionalmente se han apoyado los debates acerca de la funcionalidad de estos asentamientos. Del mismo modo paralelamente quizás sea probable redefinir estos espacios más allá de su consideración actual como meras acumulaciones de desperdicios alimenticios.

La existencia de estas evidencias debería servir, al menos, para plantear la necesidad de abordar desde otra perspectiva el análisis de las conchas halladas en los contextos arqueológicos. El empleo de metodologías adecuadas para el tratamiento de este tipo de materiales en la propia excavación, durante su almacenamiento y, lógicamente en su posterior análisis, debería posibilitar la incorporación de nuevos hallazgos instrumentales en otros contextos litorales. En este sentido, consideramos que el conocimiento global de las formaciones económico-sociales que han ex-

\footnotetext{
(12) Cuenca Solana, D. 2009. Véase nota 4.
} 
plotado el litoral podría verse beneficiada por un conocimiento más completo de todos aquellos instrumentos empleados por estos grupos de cazadores recolectores para satisfacer sus necesidades productivas.

\section{AGRADECIMIENTOS}

La Universidad de Cantabria financió esta investigación a través de una beca y un contrato predoctorales concedidos a dos de los autores (D.C.S. e I.G.Z.). Manuel R. González Morales, Alejandro García Moreno y Juan Carlos López Quintana nos ayudaron en diversas cuestiones relacionadas con este trabajo.

\section{BIBLIOGRAFÍA}

Arias Cabal, P. 1992a: "Estrategias de aprovechamiento de las materias primas líticas en la costa oriental de Asturias". En R. Mora, X. Terradas., X. Parpal y C. Plana (eds.): Tecnología y cadenas operativas líticas. Treballs d'Arqueología 1. Departament d'Història de les societats Pre-capitalistes i d'Antropologia Social. Barcelona: 37-55.

Arias Cabal, P. 1992b: "Adaptaciones al medio natural de las sociedades humanas de la región cantábrica durante el Boreal y el Atlántico". En A. Cearreta y F.M. Ugarte (eds.): The Late Quaternary in the Western Pyrenean Region. Universidad del País Vasco. Vitoria-Gasteiz: 269-283.

Banks, W. y Kay, M. 2003: "High-resolution casts for lithic use-wear analysis". Lithic Technology 28 (1): 27-34.

Binford, L. 1998: En busca del pasado. Descifrando el registro arqueológico. Crítica. Barcelona.

Bonomo, M. 2007: "El uso de los moluscos marinos por los cazadores-recolectores pampeanos". Chungara, Revista de Antropología Chilena 39 (1): 87-102.

Bonomo, M. y Aguirre, M. 2009: "Holocene Molluscs from Archaeological Sites of the Pampean Region of Argentina: Approaches to Past Human Uses". Geoarchaeology: An International Journal 24 (1): 59-85.

Choi, K. y Driwantoro, D. 2007: "Shell tool use by early members of Homo erectus in Sangiran, central Java, Indonesia: cut mark evidence". Journal of Archaeological Science 34: 48-58.

Clark, G. 1976: El Asturiense Cantábrico. Bibliotheca Prehistórica Hispana XIII, CSIC, Instituto Español de Prehistoria. Madrid.
Clemente, I. 1997: Los instrumentos líticos de Tunel VII: una aproximación etnoarqueológica. Treball d'etnoarqueología 2, CSIC-UAB. Barcelona.

Cristiani, E.; Lemorini, C.; Martini, F. y Sarti, L. 2005: "Scrappers of Callista chione from Grotta del Cavallo (Middle Paleolithic cave in Apulia): evaluating use-wear potential". En H. Luik; A. Chayke; C. Batey y L. Lougos (eds.): From hooves to horns, from mollusc, to mammoth. Manufacture and use of bone artefacts from prehistoric times to the present. Procedings of the 4th meeting of the ICAZ Worked bone Research Group at Tallin, 26th-31st of August 2003. Tallin Book Printers. Tallin: 319-324.

Dacal Moure, R. 1978: Artefactos de concha en las comunidades aborigenes cubanas. Universidad de la Habana. Cuba.

D'Errico, F. 1988:"'The use of resin replicas for the study of lithic use". En S. Olsen (ed.): Scanning Electron Microscopy in Archaeology. British Archaeological Reports, International Series 452. Oxford: 155-167.

D’Errico, F.; Giacobini, G. y Puech, P. 1982: "Varnish replicas: a new method for the study of worked bone surfaces". Ossa 9-10: 29-51.

Gibaja Bao, J.F. 1993: "El cómo y el porqué de la experimentación en análisis funcional". Revista de Arqueología 148: 10-15.

González Morales, M. 1982: El Asturiense y otras culturas locales. La explotación de las áreas litorales de la región cantábrica en los tiempos epipaleolíticos. Centro de investigación y museo de Altamira 7. Santander.

González Morales, M. 1995: "La transición del Holoceno en la Región Cantábrica: el contraste con el modelo mediterráneo español". En V. Villaverde Bonilla (ed.): Los últimos cazadores: transformaciones culturales y económicas durante el Tardiglaciar y el inicio del Holoceno en el ámbito mediterráneo. Instituto de Cultura "Juan Gil-Albert". Alicante: 63-78.

González Morales, M. R.; Straus, L.; Díez Castillo, A. y Ruiz Cobo, J. 2004: "Postglaciar Coast \& Inland: The Epipaleolithic-Mesolithic-Neolithic Transitions in the Vasco-Cantabrian Region". Munibe 56: 61-78.

González Urquijo, J. e Ibáñez Estévez, J. 1994: Metodología de análisis funcional de instrumentos tallados en sílex. Cuadernos de Arqueología 14, Universidad de Deusto. Bilbao.

Ilkjaer, J. 1979: "A new method for observation and recording of use-wear. En B. Hayden (ed.): Lithic use-wear analysis. Academic Press. New York: 345-349.

Jones O'Day, S. y Keegan, W. 2001: “Expedient Shell Tools from the Northern West Indies". Latin American Antiquity 12 (3): 274-290. 
Karali, L. 2005: "Shells from Prehistoric sites of Northern Greece". En D. Bar-Yosef (ed.): Archaeomalacology: Molluscs in former environments of human behaviour (9th Icaz Conference, Durham 2002). Oxbow Books. Oxford: 91-98.

Knutsson, K. y Hope, R. 1984: "The application of acetate peels in lithic use wear analysis". Archaeometry 26 (1): 49-61.

Lammers-Keijsers, Y. 2008: Tracing Traces from Present to past. A funtional analysis of pre-Columbian shell and stone artefacts from Anse á la Gourde and Morel, Guadaloupe, FWI. University Press. Leiden, The Netherlands.

Lindner, G. 1977: Moluscos y caracoles de los mares del mundo. Omega. Barcelona.

López Quintana, J. y Guenaga, A. 2006-2007: “Avance a la secuencia estratigráfica de la cueva de Santimamiñe (Kortezubi), tras la revisión de su depósito arqueológico en las campañas de 2004 a 2006". Krei 9: 73-103.

Maicas Ramos, R. 2008: "Objetos de concha: algo más que adornos en el Neolítico de la cuenca de Vera (Almería)". En M. Hernández Pérez; J. Soler Díaz y J. López Padilla (eds.): IV Congreso del Neolítico Peninsular. (II). (Museo Arqueológico de Alicante, 27-30 de noviembre de 2006): 313-319. Alicante.

Pascual Benito, J. 2008: "Instrumentos neolíticos sobre soporte malacológico de las comarcas centrales valencianas". En M. Hernández Pérez, J. Soler Díaz y J. López Padilla (eds.): IV Congreso del Neolítico Peninsular. (II). (Museo Arqueológico de Alicante, 27-30 de noviembre de 2006): 290-297.

Plisson, H. 1983: "An application of casting techniques for observing and recording of microwear". Lithic Technology 12 (1): 17-20.
Plisson, H. 1984: "Prise d'empreinte des surfaces osseuses: note complémentaire". Bulletin de la Société Préhistorique Française 81 (9): 267-269.

Rodríguez Rodríguez, A. y Navarro Mederos, J. 1999: "La industria malacológica de la cueva de El Tendal (San Andrés y Sauces, isla de La Palma)". Vegueta 4: 75-100.

Semenov, S. A. 1981: Tecnología prehistórica. (Estudio de las herramientas y objetos antiguos a través de las huellas de uso). Akal. Madrid.

Szabó, K. 2008: "Shell as a Raw Material: Mechanical Properties and Working Techniques in the Tropical Indo-West Pacificic". Archaeofauna 17: 125-138.

Szabó, K.; Brumm, A. y Bellwood, P. 2007: "Shell artefact production at 32.000-28.000 BP in Island Southeast Asia. Thinking across media?". Current Antropology 48 (5): 701-723.

Taborin, Y. 1974: 'La parure en coquillage de 1'Épipaléolithique au Bronce Ancien en France". Gallia Préhistoire 17: 101-179.

Toht, N. y Woods, M. 1989: "Molluscan shell knives and experimental cut-marks on bones". Journal of Field Archaeology 16: 250-255.

Vigié, B. 1987: "Essai d'étude methodologique d'outils sur coquillages de la grotte de Camprafaud (Ferrières-Poussarou, Hérault)". L'Antropologie 91 (1): 253-272.

Vigié, B. 1995: "Du déchet alimentaire à l'objet coquillier: le statut des coquillages en milieu archéologique". En G. Camps (ed.): L'Homme préhistorique et la mer. 120e Congrés National des Sociétés savantes. (Aix-en-Provence, 1995): 351-354. Paris.

Vigié, B. y Courtin, J. 1986: "Les outils sur coquilles marines dans le Neolithique du midi de la France". Mesogee 46: 51-61.

Vigié, B. y Courtin, J. 1987: "Le probleme des coquillages a bord dentele dans la prehistoire du midi de la France". Mesogee 47: 93-98. 